\title{
Antazoline for rapid termination of atrial fibrillation during ablation of accessory pathways
}

\author{
Roman Piotrowski ${ }^{1}$, Tomasz Kryński ${ }^{1}$, Jakub Baran ${ }^{1}$, \\ Piotr Futyma ${ }^{2}$, Sebastian Stec ${ }^{1}$, Piotr Kułakowski ${ }^{1}$ \\ ${ }^{1}$ Department of Cardiology, Grochowski Hospital, Postgraduate Medical School, Warsaw, Poland \\ ${ }^{2}$ Invasive Cardiology Department, St. Joseph's Centre, Rzeszow, Poland
}

\begin{abstract}
Background and aim: To assess safety and efficacy of antazoline for termination of atrial fibrillation ( $A F$ ) occurring during ablation of accessory pathways $(A P)$.

Methods: We analyzed electrophysiological mechanism of antazoline (changes in A-A interval) and the percentage of pre-excited QRS complexes before and after antazoline administration. The total dose administered and the time from the start of injection to sinus rhythm restoration were also measured.
\end{abstract}

Results: Out of consecutive 290 patients with Wolff-Parkinson-White syndrome undergoing radiofrequency $(R F)$ ablation, 12 (4.1\%) (4 females, mean age $36 \pm 20$ years) developed sustained $A F$ which did not stop spontaneously within 10 min, and antazoline in $100 \mathrm{mg}$ repeated boluses was administered. In all 12 patients the drug restored sinus rhythm after a mean of $425 \pm 365 \mathrm{~s}$ (range 43-1245 s) using a mean cumulative dose of $176 \pm 114 \mathrm{mg}$ (range $25-400 \mathrm{mg}$ ). The drug slightly prolonged $R$-R intervals during AF (from $383 \pm 106$ to $410 \pm$ $\pm 70 \mathrm{~ms}$ ) and reduced the percentage of fully pre-excited $Q R S$ complexes (from $35 \%$ to $26 \%$ ). Intracardiac recordings showed gradual increase in A-A intervals, as well as regularization and decreasing fractionation of atrial activity following drug injection (mean $A-A$ interval of $162 \pm 30$ ms at baseline $v s .226 \pm 26$ ms shortly before sinus rhythm restoration, $p<0.001$ ). AP was not completely blocked in any patient which enabled continuation of ablation.

Conclusions: Antazoline safely and rapidly converts AF into sinus rhythm during ablation of AP. The drug does not block AP completely, enabling continuation of ablation. The drug converting AF into more organized atrial activity (atrial flutter/tachycardia) before sinus rhythm resumption. (Cardiol J 2014; 21, 3: 299-303)

Key words: antazoline, accessory pathway, atrial fibrillation

\section{Introduction}

Catheter ablation is an effective and curative treatment in patients with Wolff-Parkinson-White (WPW) syndrome. Atrial fibrillation (AF) may occur during the procedure and make ablation more difficult or impossible to perform due to intermittent pre-excitation, electrode instability or inability to map atrial insertion of the accessory pathway (AP). Usually AF stops spontaneously within seconds or minutes, however, in some patients it does not self-terminate. In such cases rapid conversion to sinus rhythm (SR) may be achieved by electrical cardioversion, however, it requires general anes-

Address for correspondence: Dr Roman Piotrowski, Department of Cardiology, Grochowski Hospital, Postgraduate Medical School, ul. Grenadierów 51/59, 04-073 Warszawa, Poland, e-mail: rpiotrow@op.pl

Received: 17.06.2013 Accepted: 31.07.2013 
thesia and does not prevent from immediate $\mathrm{AF}$ recurrence. Antiarrhythmic drugs may terminate $\mathrm{AF}$, however, they can also temporarily block $\mathrm{AP}$ and preclude ablation [1]. Therefore, a rapidly acting drug that terminates $\mathrm{AF}$ and does not totally block AP would be very useful.

Antazoline is an antihistaminic agent with antiarrhythmic quinidine-like properties [2-4]. In some countries it is registered for intravenous termination of cardiac arrhythmias and has been successfully used for many years for rapid termination of AF [5-8]. However, due to the lack of large randomized trials the drug is not listed in any formal guidelines. The aim of our study was to assess safety and efficacy of antazoline for termination of $\mathrm{AF}$ occurring during ablation of AP.

\section{Methods}

Out of consecutive 290 patients with WPW syndrome undergoing radiofrequency ablation in 2 institutions, 12 (4.1\%) (4 females, mean age $36 \pm$ \pm 20 years) developed $\mathrm{AF}$ which did not stop spontaneously or after Valsalva maneuver during $10 \mathrm{~min}$ and an operator decided that antazoline should be administered in order to restore SR. From the database, we collected demographic and clinical data, as well as electrophysiological parameters. The off-line analysis of intracardiac electrocardiograms (ECG) were performed to analyze atrial activity, ventricular response during $\mathrm{AF}$, and the percentage of pre-excited QRS complexes before and after antazoline administration. The total dose administered and the time from the start of injection to sinus rhythm restoration was examined.

Antazoline was given intravenously in boluses of $100 \mathrm{mg}$ injected over $2-3 \mathrm{~min}$ periods with $1 \mathrm{~min}$ interval between boluses. The maximal cumulative dose that could have been administered was $400 \mathrm{mg}$. A 12-lead ECG and intracardiac recordings, as well as blood pressure were continuously monitored. The drug was stopped immediately after SR restoration or when the maximal allowed cumulative dose was given. Antazoline was contraindicated when a patient had severe underlying cardiac disorder defined as heart failure symptoms in NYHA class > II or markedly reduced left ventricular ejection fraction $<40 \%$. Also significant hypotension (systolic blood pressure $<90 \mathrm{~mm} \mathrm{Hg}$ ) was a contraindication since the drug may cause hypotension.

Electrocardiographic measurements were obtained at baseline and after antazoline administration, following each $100 \mathrm{mg}$ bolus and just before termination of AF. Measurements were performed using electronic calipers from the tracings recorded in the electrophysiological system at a speed of $100 \mathrm{~mm} / \mathrm{s}$. During AF, the shortest pre-excited $\mathrm{R}-\mathrm{R}$ interval was measured in the lead with the most prominent pre-excitation. The percentage of pre-excited QRS complexes was calculated from a $30 \mathrm{~s}$ continuous ECG tracing by dividing the number of pre-excited beats and total number of beats during this period. Intracardiac atrial activity was analyzed from a diagnostic catheter introduced into coronary sinus. We measured duration of A-A intervals (consecutive atrial cycles during $30 \mathrm{~s}$ ) and variability of A-A intervals (standard deviation [SD] of consecutive A-A intervals during $30 \mathrm{~s}$ ).

All patients gave informed written consent to undergo catheter ablation and to use their demographic, clinical and electrocardiographic data in further research. Catheter ablation was performed using standard methods. The patients were followed in the outpatient clinic to assess the long-term effect of the procedure.

\section{Statistical analysis}

The results are presented as mean \pm SD or numbers and percentages. Numerical variables were compared using 2 -sided paired t-test and qualitative variables - using $\chi^{2}$ test with Yates correction or Fisher's exact test where appropriate. A p value $<0.05$ was considered significant.

\section{Results}

Demographic and clinical characteristics of the studied patients are presented in Table 1. Antazoline converted AF into SR in all patients. The drug was well tolerated and no side effects were noted. The mean effective cumulative dose

Table 1. Demographic and clinical characteristics of studied patients.

\begin{tabular}{lc}
\hline Age [years] & $36 \pm 20$ \\
Male gender & $8(67 \%)$ \\
History of palpitations & $11(92 \%)$ \\
History of documented atrial fibrillation & $3(25 \%)$ \\
Overt pre-excitation & $9(75 \%)$ \\
Concomitant disorders & \\
Arterial hypertension & $1(8 \%)$ \\
Coronary arterial disease & $1(8 \%)$ \\
Heart failure & $0(0 \%)$ \\
Diabetes mellitus & $1(8 \%)$ \\
\hline
\end{tabular}


Table 2. Effects of antazoline on electrocardiological and electrophysiological parameters during atrial fibrillation.

\begin{tabular}{lccc}
\hline Parameter & Before antazoline & After antazoline* & P \\
\hline Shortest R-R interval [ms] & $383 \pm 106$ & $410 \pm 70$ & NS \\
Percent of pre-excited QRS complexes & 35 & 26 & NS \\
Mean A-A interval [ms] & $162 \pm 30$ & $227 \pm 26$ & 0.001 \\
Variability of A-A intervals [ms] & 30 & 26 & NS \\
\hline
\end{tabular}

*Total cumulative dose

of antazoline was $176 \pm 114 \mathrm{mg}$ (range $25-400 \mathrm{mg}$ ) and the mean time to conversion $-425 \pm 365 \mathrm{~s}$ (range $43-1245 \mathrm{~s}$ ).

The effects of antazoline on electrocardiographic and electrophysiological parameters are presented in Table 2 and Figures 1 and 2 . The drug slightly prolonged $\mathrm{R}-\mathrm{R}$ intervals during $\mathrm{AF}$ and tended to reduce the percentage of pre-excited QRS complexes. Intracardiac recordings showed gradual significant increase in A-A intervals and regularization of atrial activity following drug injection.

Although the drug reduced the degree of pre-excitation, AP was not completely blocked in any patient which enabled continuation of ablation, both in patients with overt WPW and in those with concealed AP. No further AF recurrences during ablation were noted. All procedures were effective and there were no recurrences of pre-excitation or palpitations during follow-up period (mean $29 \pm 26$ months, range 4-93 months).

\section{Discussion}

The main findings of the present study are that antazoline effectively and rapidly converts AF into SR during ablation of AP. Although the drug prolongs refractory period of AP, it does not block AP completely, enabling continuation of ablation. The study also shows that antazoline is safe in termination of AF in WPW patients.

When AF develops during ablation of AP, it may complicate continuation of the procedure. The catheter is less stable and the pre-excitation during $\mathrm{AF}$ may be variable with some beats completely without pre-excitation. Although in some patients with overt pre-excitation the procedure may be continued during $\mathrm{AF}$ and effective $\mathrm{AP}$ conduction abolition may be achieved, applications delivered during SR are more accurate and probably more effective. Moreover, in patients with concealed AP, AF precludes effective procedure because the atrial insertion of AP cannot be identified. Thus, prompt and durable restoration of SR is desired in the majority of patients.

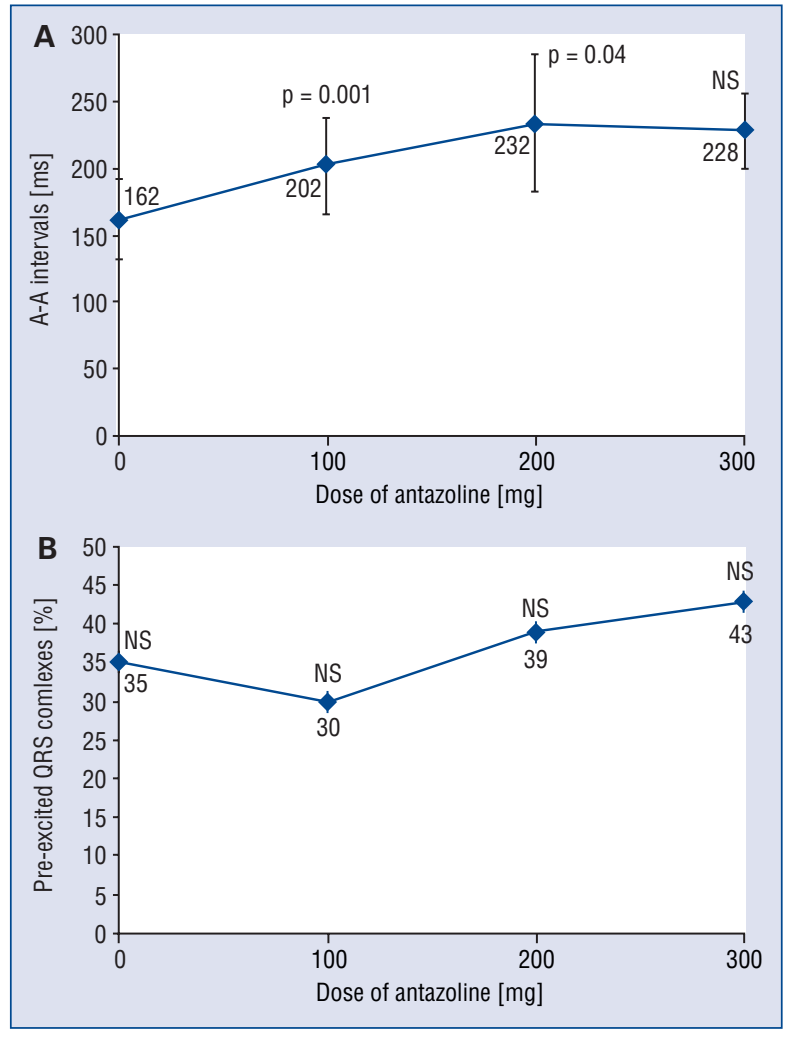

Figure 1. Changes in analyzed parameters following consecutive doses of antazoline; A. Mean A-A intervals; B. Percent of pre-excited QRS complexes.

Antazoline has been used for rapid AF termination in some countries for many years, however, due to the lack of large, randomized studies it is not mentioned in the guidelines as the agent recommended for AF termination. There is an ongoing trial which will establish the role of antazoline in this setting [9]. The drug has quinidine-like antiarrhythmic properties, including prolongation of conduction and refractoriness. It has a relatively short half-life time of $3 \mathrm{~h}$ and electrophysiological effects disappear after $1 \mathrm{~h} \mathrm{[3]} \mathrm{which} \mathrm{is} \mathrm{especially}$ important when only short-lasting and transient changes in electrophysiological parameters are 


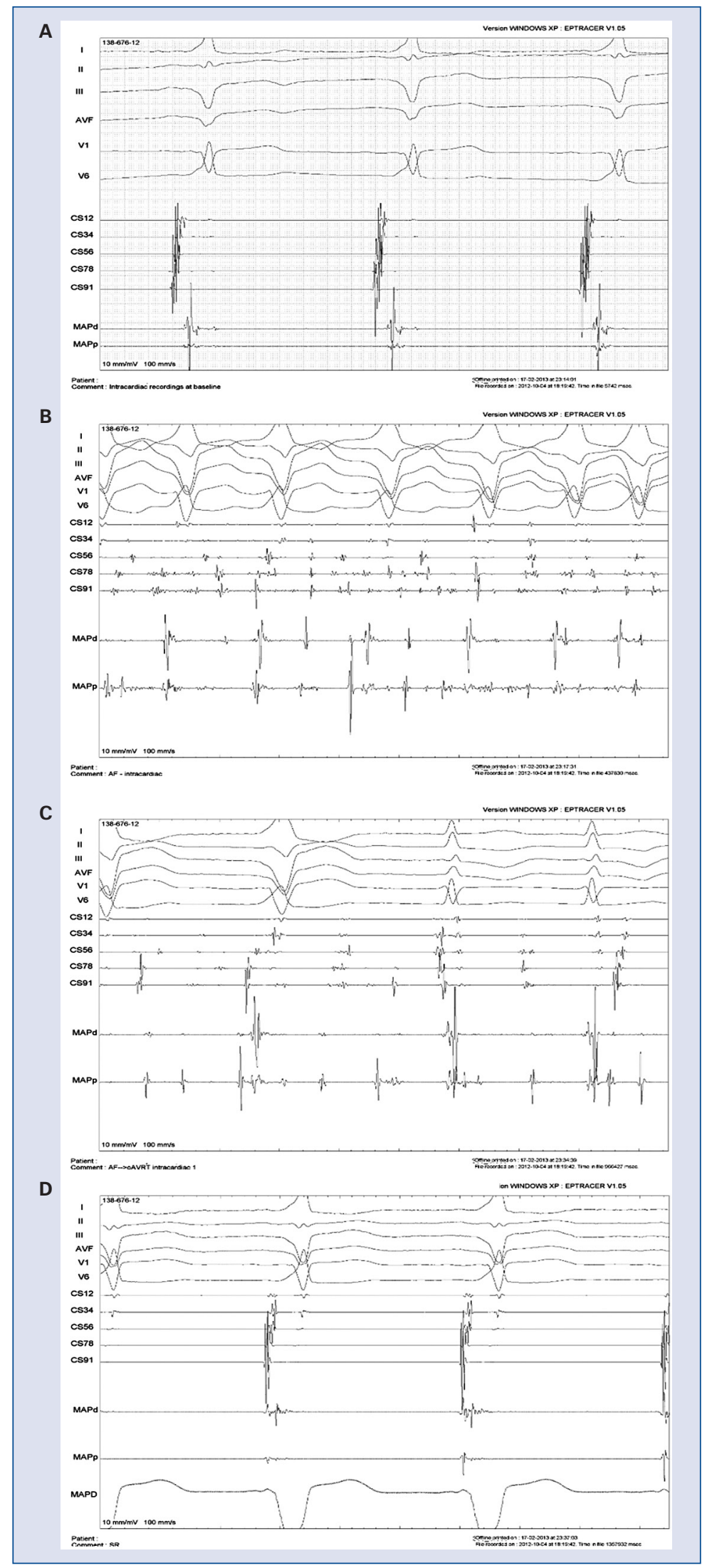

Figure 2. Intracardiac recordings at baseline (A), at the onset of atrial fibrillation (B), after antazoline administration shortly before atrial fibrillation termination (C) and after restoration of sinus rhythm (D). In panels $B$ and $C$ gradual lengthening of A-A intervals is seen; CS — diagnostic catheter placed in coronary sinus; MAP — ablation catheter, I, II, III, aVF, V $1, \mathrm{~V}_{6}$ - surface ECG. 
required. The drug acts rapidly, terminating $\mathrm{AF}$ within 10-20 min from the start of injection [7]. Observational studies showed that overall efficacy of the drug in short-lasting $\mathrm{AF}$ ranges between $6 \%$ and $81 \%$ [2, 7], exceeding $50 \%$ in the largest reported cohort of 1325 patients [8]. One study showed that antazoline was completely ineffective in termination of permanent $\mathrm{AF}$ [10].

The above-mentioned antazoline properties prompted its usage during electrophysiological procedures in some laboratories. We also have been successfully using antazoline for a long-time not only in the electrophysiology laboratory but also in the emergency department or cardiac intensive care unit. The drug is contradicted in patients with severe heart failure or markedly reduced left ventricular ejection fraction due to modest negative inotropic effect and also because there is usually transient increase in ventricular response during $\mathrm{AF}$ following drug administration which may be dangerous in patients with severe cardiac disease. The latter effect is probably due to the gradual reduction of the number of fibrillatory waves in the atrium, resulting in less concealed conduction in the atrio-ventricular node which promotes conduction from the atria to the ventricles and fastens ventricular rate. This phenomenon is similar to that seen following administration of other antiarrhythmic agents such as quinidine, ajmaline and flecainide or propafenone.

Our results showed that antazoline not only terminated AF but also prolonged probably refractoriness of the AP, seen as the reduction of the percentage of the pre-excited QRS complex during AF. While such an effect is desirable during any chronic antiarrhythmic therapy in a patient with WPW syndrome, it is unwanted during ablation because drug-induced block in the AP usually precludes proper mapping and ablation of AP. However, in all our patients antazoline did not completely block the AP and all procedures were successfully completed. Thus, we believe that antazoline can be used in this situation instead of electrical cardioversion which requires anesthesia and does not prevent from AF recurrence, or other antiarrhythmic drugs such as flecainide or propafenone which may block AP completely and have slower onset of action and longer duration of electrophysiological effects on AP.

Another advantage of using antazoline is its very low price (currently 1 euro per $200 \mathrm{mg}$ dose) which is much less than the cost of another antiarrhythmic drug such as propafenone or vernakalant, used in this setting.

\section{Limitations of the study}

This was a retrospective analysis of our database and the number of patients was small. The study was not randomized and not controlled by placebo. We did not perform systematically programmed atrial and ventricular stimulation following antazoline administration and $\mathrm{AF}$ termination, thus, detailed effects of the drug on the conduction and refractoriness of the atrio-ventricular node and $\mathrm{AP}$ were not examined. We did not measure drug plasma levels, thus, correlation between drug concentration, effectiveness in AF termination, and effects on electrophysiological properties of AP and atrio-ventricular node could not be examined.

\section{Conclusions}

Antazoline safely and rapidly converts AF into SR during ablation of AP. The drug does not block AP completely, enabling continuation of ablation. The drug gradually increases A-A intervals during $\mathrm{AF}$ converting it into more organized atrial activity (atrial flutter/tachycardia) before SR resumption. The on-going multicenter randomized studies will establish the role of antazoline in acute AF termination.

\section{Conflict of interest: none declared}

\section{References}

1. Fauchier JP, Cosnay P, Rouesnel P et al. Effects of oral and injectable flecainide in patients with an accessory atrioventricular pathway. Arch Mal Coeur Vaiss, 1985; 78: 81-90.

2. Dreifus L, McGarry T, Watanabe Y. Clinical and physiologic effects of antazoline a new antiarrhythmic agent. Heart J, 1963; 65: 607.

3. Kline RS, Dreifus LS, Watanabe Y et al. Evaluation of the antiarrhythmic properties of antazoline. Am J Cardiol, 1962; 9: 564-567.

4. Reynolds EW, Baird WM, Clifford ME. A clinical trial of antazoline in the treatment of arrhythmias. Am J Cardiol, 1964; 14: 513-521.

5. Gehring DA, Kehler JG. Conversion of atrial fibrillation with antazoline hydrochloride. Angiology, 1970; 21: 11-17.

6. Antiani JA. A clinical evaluation of antazoline in cardiac arrhythmia. Indian Heart J, 1971; 23: 212.

7. Srzednicki M, Sadowski Z, Kulikowski A. Evaluation of the anti-arrhythmia effectiveness of Phenazolinum Polfa in paroxysmal atrial fibrillation. Pol Tyg Lek, 1990; 45: 924-927.

8. Kuch M, Janiszewski M, Dłużniewski M, Mamcarz A. Antazolina: Lek nieskuteczny czy niedoceniony w leczeniu napadowego migotania przedsionków? Pol Przegl Kardiol, 2000; 2: 247-251.

9. Farkowski MM, Maciąg A, Dąbrowski R, Pytkowski M, Kowalik I, Szwed H. Clinical efficacy of antazoline in rapid cardioversion of paroxysmal atrial fibrillation: A protocol of a single center, randomized, double-blind, placebo-controlled study (the AnPAF Study). Trials, 2012; 13: 162; doi:10.1186/1745-6215-13-162.

10. Shah SS, Vaidya $\mathrm{CH}$. Antazoline in the treatment of cardiac arrhythmias. Postgrad Med J, 1972; 48: 304-307. 